\title{
TOWARDS A BROADER THEORY OF MOBILE PROCESSES
}

\author{
Robin Milner \\ University of Cambridge, Cambridge, $U K$
}

Bigraphs are a topographical model of reactive systems that aim to unify existing theoretical approaches to mobile communicating agents. They combine two structures orthogonally: connectivity and locality. Thus, for example, they represent both ambients and pi-calculus; the topography deals not only with (even physical) locality but also with abstract notions such as the scope of a name. In my talk I shall explain how recent joint work with Jamey Leifer on relative pushouts enables transition systems to be derived for pi-calculus and ambients (in recent work by Ole Jensen), and I shall present condition-event Petri nets as an example. 